\title{
Life and Evolution in terms of maximum entropy production principle
}

\author{
Leonid M. Martyushev ${ }^{1,2}$, \\ ${ }^{1}$ Ural Federal University, Mira St. 19, 620002 Ekaterinburg, Russia; \\ ${ }^{2}$ Institute of Industrial Ecology, S Kovalevskoi St. 20a, 620219 Ekaterinburg, Russia \\ leonidmartyushev@gmail.com
}

\begin{abstract}
Maximum entropy production principle (MEPP) has been formulated in the mid-twentieth century, and today it has acquired the status of an important principle of science, which is extremely effective in considering various non-equilibrium problems. In this study, for the first time, definition of life is based on an easily measurable physical quantity that is entropy production. Life and evolution are discussed from the point of view of MEPP and the Universe, but not a human.
\end{abstract}

PACS numbers: 05.70.Ln, 87.23.-n

\section{Introduction}

Maximum entropy production principle (MEPP) is an important principle of modern science that has proven itself well in the analysis and modeling of various non-equilibrium processes [1-7]. The problems of evolution (not only biological, but also cosmic, technological, etc.) from the point of view of MEPP or closely related principles are considered quite fully in the following review papers [6,8-20]. Despite that this principle has existed in science for a long time, there is no discussion of some important questions of evolutionary biology, including those related to the definition of life in the MEPP literature. This short note will address these issues.

\section{Results and Discussion}

According to the generalized formulation of MEPP: at each level of description, with preset external constraints, a local relationship between the cause and the response of a non-equilibrium system is established in order to maximize the specific entropy production or, quite briefly, Nature in its development prefers systems that produce more and more specific entropy [6] ${ }^{1}$. The consequence of this principle is the emergence of life on Earth, the complexity of living beings in the course of evolution, the appearance of man and the entire course of development of our civilization (from the man who learned to use fire, to the widespread use of oil and nuclear energy $)^{2}$. As a result, MEPP turns out to be the most important principle explaining the direction (progressiveness) of biological and technological evolution. According to this principle, the complexity in the process of evolution is the result of the fact that in the world far from equilibrium, systems (cells, organs, organisms, communities, etc.) arise with more and more entropy production. Increase of entropy production is both the source for the emergence of "biological material" and, in many cases, the cornerstone of natural selection. So, MEPP allows to combine in a very original way both the idea of J. Lamarck about "striving for perfection", and opposed to it the idea of natural selection of C. Darwin. Following and "being selected" according to MEPP, organisms naturally become more complex, since the large organization and complexity requires the consumption of more free energy, which emits more entropy during processing.

The described complications during evolution (usually called progressive) are a rather rare evolutionary events. The frequency of such transitions is significantly lower than the frequency of

\footnotetext{
${ }^{1}$ In many cases, we can talk not about the production of entropy, but of heat.

${ }^{2}$ Indeed, all of the above occurs with an increasing specific release of entropy (heat); numerous confirmations of this can be found in the Refs. [10,12,13,17].
} 
transformations occurring at the same level of achieved complexity. Transitions to the next evolutionary level (the establishment of new relationships between driving forces and emerging functions) take place according to MEPP. However, at achieved stage of the system's development, long-term system optimization (through competition and selection) is possible, including slightly decreasing entropy production (for systems which are not too far from equilibrium). The following example can be presented. Humankind, developing, began to use electricity on a massive basis. That results to an abrupt increase in heat generation. However, work for more efficient (economical) production and consumption of electricity began immediately. Obviously, this do not return humankind to the previous level of heat generation and is only a small "stop" of civilization before a new energy leap.

MEPP allows to look at issues related to the progress and future of humankind from a new, somewhat unexpected side. So, currently, due to climate warming, activities being planned or are already being taken to limit heat generation and greenhouse gas emissions. In this planning, MEPP is not currently taken into account. However, without due consideration of this principle, measures taken to combat climate change may be insufficient or even dangerous for the future of humankind. Indeed, Nature is guided by the underlying principles, including MEPP. The logic of people who cares about preserving their living environment is obviously different. If we restrict ourselves in the processing of available energy, will we not come into conflict with Nature, which created us? In this case, do not Nature choose systems that are more evolutionarily progressive from its point of view, having displaced us from the top onto which it placed us? These new systems will produce more heat using all available energy. We are used to looking at the world around us, putting ourselves and our needs at its center, but if you look from the point of view of the laws of Nature, the Universe ... As a result, caring for our environment, "preserving" it for future generations, do we not fall into the trap leading to the death of human race?

Using MEPP, one can answer the most important question of biology: what is life? As known, this issue has been discussed many times and does not have a generally accepted solution. According to [21], there are more than a hundred definitions. So, according to the definition that NASA proposed in 1994 with the goal of finding life in the Universe [22]: "life is a self-sustaining chemical system capable of Darwinian evolution". However, later this definition was criticized. One of the shortest definitions of life is self-reproduction with variations [21]. All currently available definitions of life operate with concepts that are often difficult to define strictly and, moreover, measure accurately ${ }^{3}$. This creates enormous difficulties in the tasks of searching for extraterrestrial life. Another common important drawback of existing definitions is that these definitions try to endow the concept of "life" with qualities that this or that researcher considers the most important for life, but at the same time this researcher does not try to look at himself from the position of Nature and its laws, but takes position related to close and understandable "living" organisms. As a result, such concepts in the definitions of life as: DNA, protein, carbon, water, metabolism, mutations, reproduction, etc. appear. We should not define our own kind from our subjective point of view and assign them the title "life". However, when we "search" for life, we must search systems that are at the same stage of development with us in terms of the Universe and its laws of development. Just then, when defining life, we will be objective and get rid of our ego-/anthro- centrism. This is what MEPP allows. From the point of view of MEPP and based on the results of Refs. [6, 10-14], the following definition can be proposed as a hypothesis: Life is a region of space-time with values of specific entropy production in the range of values from $10^{3}$ to $10^{5}$ specific entropy production of the star near which this region is located.

\section{Conclusions}

Many properties of life can be derived from MEPP and the proposed definition, in particular: nonequilibrium process, stability, emergence, and ability to evolve. However, this is a topic for a special paper. We note only three important points.

\footnotetext{
${ }^{3}$ For example, such concepts as "complexity", "self-reproduction with changes", self-sustaining", etc.
} 
(1) Specific entropy production is calculated on volume. Based on the data of [23] (see also $[10,12,13])$, mean metabolic rates of living matter is approximately in the range from 0.1 to 10 $\mathrm{W} / \mathrm{kg}$, which for standard conditions and quasi-stationary processes corresponds to the specific production of entropy in the range from 0.1 to $10 \mathrm{~W} /\left(\mathrm{m}^{3} \mathrm{~K}\right.$ ) (in order of magnitude). The specific entropy production of the Sun is of the order of $10^{-4} \mathrm{~W} /\left(\mathrm{m}^{3} \mathrm{~K}\right)$ [24-26]. As a result, the value given in the above definition is obtained. It is important here that the volume-specific entropy production of main sequence stars is equal to the solar with good accuracy [24-26]. Therefore, volume-specific entropy production is quite universal for the overwhelming number of stars (note that the massspecific entropy production varies greatly for main sequence stars) [26]. The consideration of specific production with respect to volume rather than mass is also associated with the fact that volume is a quantitative characteristic (measure) of space, and mass is a characteristic of matterenergy in space. An additional important reason for this choice is the simplicity of measuring volume rather than mass in astronomical investigations.

(2) The region that appears in the definition cannot be homogeneous (but, for example, mosaic) relative to the values of the specific production of entropy. Over time, these maximum values, according to the MEPP, will increase.

(3) The proposed definition is not suitable for the next level of organization of the living (social, technological, etc.), in which energy sources began to be used outside the body (for example, fire, power plants, etc.). It is not difficult to define such systems by analogy with the above definition of life, using, for example, the heat production data contained in Refs. [12, 13].

\section{References}

1) Ziegler $\mathrm{H}$ An Introduction to Thermomechanics (Amsterdam: North-Holland, 1983)

2) Ozawa H, et al Rev. Geophys. 411018 (2003)

3) Kleidon A, Lorenz R D (Eds) Non-equilibrium Thermodynamics and the Production of Entropy in Life, Earth, and Beyond (Heidelberg: Springer, 2004).

4) Martyushev L M, Seleznev V D Phys. Rep. 4261 (2006)

5) Kleidon A, Malhi Y, Cox P.M. (Eds) Maximum entropy production in ecological and environmental systems: applications and implications. Phil. Trans. R. Soc. B. 3651545 (2010).

6) Martyushev L M Entropy 151152 (2013)

7) Dewar R C, et al (Eds) Beyond the Second Law. Entropy Production and Non-equilibrium Systems (Berlin, Heidelberg: Springer-Verlag, 2014)

8) Vallino J J, Christopher K A Annu. Rev. Mar. Sci. 8333 (2016)

9) Ulanowicz R E, Hannon B M Proc. R. Soc. Lond. B 232181 (1987)

10) Zotin A.A., Lamprecht I., Zotin A.I. J. Non-Equilib. Therm. 26(1) 191 (2001)

11) Lineweaver $\mathrm{C} \mathrm{H}$ in Non-equilibrium Thermodynamics and the Production of Entropy, (Eds. Kleidon A, Lorenz R D) (Berlin, Heidelberg: Springer-Verlag, 2005) p. 67

12) Chaisson E J Complexity 16(3) 27 (2010)

13) Chaisson E J Complexity 17(1) 44 (2011).

14) Lineweaver $\mathrm{C} \mathrm{H}$ in Beyond the Second Law-Entropy Production and Non-Equilibrium Systems (Eds R C Dewar, et al) (Berlin, Heidelberg: Springer, 2014), p. 415

15) Skene K R Entropy 175522 (2015)

16) Sawada Y, Daigaku Y, Toma K arXiv:2003.11779v1 (2020)

17) Frank A, Kleidon A, Alberti M Anthropocene 1913 (2017)

18) Swenson R Syst. Research 6(3) 187-197 (1989).

19) Michaelian K Earth Syst. Dynam. 237 (2011)

20) Herrmann-Pillath C Ecolog. Econom. 119432 (2015)

21) Trifonov E N J. Biomol. Struct. Dynam. 29(2) 259 (2011) 
22) Benner S A Astrobiology 10(10) 1021 (2010)

23) Makarieva A M, Gorshkov V G, Li B-L, et al PNAS 105(44) 16994 (2008)

24) Martyushev L M, Zubarev S N Entropy 17658 (2015)

25) Martyushev L M, Zubarev S N Entropy 173645 (2015)

26) Martyushev L M, Zubarev S.N. Physica A 528121403 (2019) 